\title{
ISOCAM and molecular observations of the edge of the Horsehead nebula
}

\author{
A. Abergel ${ }^{1}$, D. Teyssier ${ }^{2,13}$, J. P. Bernard ${ }^{1}$, F. Boulanger ${ }^{1}$, A. Coulais ${ }^{12}$, D. Fosse ${ }^{2}$, E. Falgarone ${ }^{2}$, M. Gerin ${ }^{2}$, \\ M. Perault ${ }^{2}$, J.-L. Puget ${ }^{1}$, L. Nordh ${ }^{3}$, G. Olofsson ${ }^{3}$, M. Huldtgren ${ }^{3}$, A. A. Kaas ${ }^{3}$, P. André ${ }^{4}$, S. Bontemps ${ }^{9}$, \\ M. M. Casali ${ }^{10}$, C. J. Cesarsky ${ }^{11}$, E. Copet ${ }^{5}$, J. Davies ${ }^{6}$, T. Montmerle ${ }^{4}$, P. Persi ${ }^{7}$, and F. Sibille ${ }^{8}$ \\ ${ }^{1}$ Institut d'Astrophysique Spatiale, Université Paris-Sud, Bât. 121, 91405 Orsay, France \\ 2 LERMA, École Normale Supérieure et Observatoire de Paris, 24 rue Lhomond, 75231 Paris Cedex 05, France \\ 3 Stockholm Observatory, 13336 Saltsjöbaden, Sweden \\ ${ }^{4}$ Service d'Astrophysique, Centre d'Études de Saclay, 91191 Gif-Sur-Yvette Cedex, France \\ 5 LESIA, Observatoire de Paris-Meudon, 5 place Jules Janssen, 92195 Meudon Cedex, France \\ 6 Joint Astronomy Center, 660 N. A'Ohoku Place, University Park, Hilo, HI 96720, USA \\ 7 Istituto Astrofisica Spaziale, Area di Ricerca Tor Vergata, via del Fosso del Cavaliere, 00133 Roma, Italy \\ 8 Observatoire de Lyon, 69230 Saint Genis Laval, France \\ 9 Observatoire de Bordeaux, BP 89, 33270 Floirac, France \\ 10 Royal Observatory, Blackford Hill, Edinburgh, UK \\ 11 ESO, Headquarters, Karl-Schwarzschild-Strasse 2, 85748 Garching, Germany \\ 12 LERMA, Observatoire de Paris, 61 Av. de l'Observatoire, 75014 Paris, France \\ 13 SRON - Low Energy Astrophysics Division Landleven 12, 9747 AD Groningen, The Netherlands
}

Received 2 August 2002 / Accepted 28 May 2003

\begin{abstract}
We present ISOCAM observations $(5-18 \mu \mathrm{m})$ of the Horsehead nebula, together with observations of the $(J=1-0)$ and $(J=2-1)$ transitions of ${ }^{12} \mathrm{CO},{ }^{13} \mathrm{CO}$ and $\mathrm{C}^{18} \mathrm{O}$ taken at the IRAM 30-m telescope.

The Horsehead nebula presents a typical photodissociation region illuminated by the $09.5 \mathrm{~V}$ system $\sigma$ Ori. The ISOCAM emission is due to very small particles transiently heated to high temperature each time they absorb a UV photon. A very sharp filament (width: $\sim 10^{\prime \prime}$ or $\sim 0.02 \mathrm{pc}$ ) is detected by ISOCAM at the illuminated edge of the nebula. This filament is due to a combined effect of steep increase of the column density and extinction of incident radiation, on typical sizes below $\sim 0.01 \mathrm{pc}$. Both the three-dimensional shape and the local density of the illuminated interface are strongly constrained. The dense material forming the edge of the Horsehead nebula appears illuminated edge-on by $\sigma$ Ori, and the particles located beyond the border should not be affected by the incident radiation field. This structure may be due to dense filaments in the parental cloud which have shielded the material located in their shadow from the photo-dissociating radiations.

The measurement of the penetration depth of the incident radiation from the infrared data $(\sim 0.01 \mathrm{pc})$ gives a density of a few $10^{4} \mathrm{~cm}^{-3}$ just behind the bright filament. This value is comparable to the estimate of the density beyond the edge and deduced from our molecular observations, and also to the density behind the ionization front calculated in the stationary case. The material behind the illuminated edge could also be non-homogeneous, with clump sizes significantly smaller than the observed penetration depth of $\sim 0.01 \mathrm{pc}$. In that case no upper limit on the average density just behind the illuminated edge can be given.
\end{abstract}

Key words. ISM: individual objects: Horsehead - ISM: dust, extinction - ISM: clouds - infrared: ISM - radio lines: ISM

\section{Introduction}

Most of the molecular material of our Galaxy is located in regions where UV radiation controls the molecular content and the thermal balance. Molecular clouds in the vicinity of a bright star develop photodissociation regions (PDRs) which are unique targets to investigate this interaction of molecular matter with starlight.

The observations of PDRs in atomic and molecular emission lines are generally conducted at angular resolutions larger

Send offprint requests to: A. Abergel, e-mail: abergel@ias.fr than $\sim 1^{\prime}$ (or $0.1 \mathrm{pc}$ at a distance of $400 \mathrm{pc}$ ), which do not allow a detailed analysis of the illuminated interfaces. However, they reveal a strong clumping of the emitting gas (see the review of Hollenbach \& Tielens 1997). The observations of atomic lines ([OI], $[\mathrm{CII}]$, and $[\mathrm{CI}]$ ) indicate emission scales much larger than expected for homogeneous material. The emission of many molecular species (e.g. ${ }^{13} \mathrm{CO}, \mathrm{C}^{18} \mathrm{O}, \mathrm{CS}$ ) appears to originate essentially from clumps $\left(n \sim 10^{4} \mathrm{~cm}^{-3}\right)$, while the detection of emission due to molecules with high critical densities (e.g. $\mathrm{HCO}^{+}$, high level transitions of CS) reveals the presence of high-density molecular gas $\left(n>10^{5} \mathrm{~cm}^{-3}\right)$. Molecular 
observations at higher angular resolution (10-20") have also been conducted for very excited PDRs, such as the Orion bar (van der Werf et al. 1996). High density clumps are detected, with size in the range from 0.02 to $0.1 \mathrm{pc}$. Recently, the 1-0 $\mathrm{S}(1)$ fluorescent line of $\mathrm{H}_{2}$ in the reflexion nebula NGC 2023 has been mapped by Field et al. (1998) \& Mc Cartney et al. (1999) at an angular resolution of $\sim 1^{\prime \prime}(\sim 2 \times$ $\left.10^{-3} \mathrm{pc}\right)$. The images reveal filamentary emission interpreted as evidence for density contrasts on a milliparsec scale. The presence in PDRs of density contrasts down to such small scales would dramatically affect the chemical structure and the energy balance, by permitting the radiation to penetrate deeper into the cloud.

This paper presents ISOCAM observations (Cesarsky et al. 1996) of the Horsehead nebula, together with observations of the $(J=1-0)$ and $(J=2-1)$ transitions of ${ }^{12} \mathrm{CO},{ }^{13} \mathrm{CO}$ and $\mathrm{C}^{18} \mathrm{O}$ taken at the IRAM 30-m telescope. The edge of the Horsehead nebula is illuminated edge-on by the $09.5 \mathrm{~V}$ system $\sigma$ Ori and is ideal to study the penetration of radiation and the density structure of a PDR. The ISOCAM observations are taken from the large scale mapping of the southern part of Orion B conducted in the two broad-band filters LW2 and LW3 (5-8.5 $\mu \mathrm{m}$ and $12-18 \mu \mathrm{m}$ respectively) and presented in Abergel et al. (1999, 2002). The emission detected in the LW2 filter is dominated by the aromatic features around $7.7 \mu \mathrm{m}$, while the emission in the LW3 filter is continuum-like (Abergel et al. 2002). In the interstellar medium, the intensity of the aromatic bands is found to scale with the UV radiation field (for $\chi$ ranging between $\sim 1$ and $\sim 10^{4}$, where $\chi$ is the intensity of the UV radiation field normalised to the local interstellar radiation field, see Habing 1968), while the shape and relative amplitude of their profiles appear remarkably constant (Boulanger et al. 1998; Uchida et al. 2000). This is expected for emission from very small particles transiently heated to high temperature each time they absorb a UV photon (Léger \& Puget 1984; Sellgren et al. 1985).

The angular resolution of the ISOCAM observations (6") is $\sim 10$ times better than previous infrared or molecular and atomic lines observations conducted in this region. Thus, unprecedented constraints both on the shape of the illuminated interface and the penetration of the incident radiation can be derived. The paper is organised as follows. We present the Horsehead nebula in Sect. 2. The ISOCAM and molecular observations are described in Sects. 3 and 4, respectively. We show in Sect. 5 that these observations bring new constraints on the three-dimensional shape, the local density and the clumpiness of the material at the illuminated edge of the nebula. The main conclusions are given in the last section.

\section{Presentation of the Horsehead nebula}

In front of the western illuminated edge of the molecular cloud L1630, the visible plates are dominated by extended red emission due to $\mathrm{H} \alpha$ emission line emerging from the HII region IC 434 (e.g. Malin 1987). In the visible, the Horsehead nebula (B33 in the catalogue of Barnard 1913) emerges from the edge of L1630 as a dark cloud in the near side of IC 434 (Fig. 1). L1630 is located at a distance of $400 \mathrm{pc}$ (from the study of the distances to B stars in the Orion association by Anthony-Twarog 1982). At this distance, 1' corresponds to $0.12 \mathrm{pc}$.

At first one expects the two OB-systems $\zeta$ Ori to the north and $\sigma$ Ori to the west to illuminate the western edge of L1630 (Reipurth \& Bouchet 1984). But, as suggested by Malin (1987), $\zeta$ Ori is located closer than $\sigma$ Ori, which has been confirmed by Hipparcos estimates of the distance (Perryman et al. 1997): $352 \pm 113 \mathrm{pc}$ for $\sigma$ Ori, $250 \pm 50 \mathrm{pc}$ for $\zeta$ Ori. Located at a projected distance of $\sim 0.5^{\circ}$ (or $3.5 \mathrm{pc}$ ) from the Horsehead nebula, $\sigma$ Ori is a O9.5 V binary system (Warren \& Hesser 1977), with an effective temperature of $\sim 33000 \mathrm{~K}$ (Panagia 1973). Assuming that $\sigma$ Ori and the Horsehead nebula are in a common plane perpendicular to the line of sight, the far-UV intensity of the incident radiation field illuminating the Horsehead nebula is $\sim 100$, which is moderate compared to those of classical PDRs illuminated by $\mathrm{O}$ stars (generally $\chi \sim 10^{4}-10^{5}$, e.g. Tielens et al. 1993). For such a low intensity, the ISOCAM emission is due to transiently heated particles, and scales with the incident radiation field.

The Horsehead nebula coincides with a CS clump (Lada et al. 1991; Zhou et al. 1993). From multi-transition CS observations (resolution: $11-24^{\prime \prime}$ or $0.02-0.04 \mathrm{pc}$ ) and using a Large Velocity Gradient model, Zhou et al. have derived a density of $\sim 2 \times 10^{4} \mathrm{~cm}^{-3}$. A local density of $3 \times 10^{4} \mathrm{~cm}^{-3}$ is also found by Kramer et al. (1996) from ${ }^{12} \mathrm{CO}$ and ${ }^{13} \mathrm{CO}$ observations taken with a resolution of $1.5-2^{\prime}$ (or $\sim 0.2 \mathrm{pc}$ ). On the other hand, the penetration depth of UV radiation measured by Zhou et al. (1993) using the spatial extent of the [CII] $158 \mu \mathrm{m}$ emission (resolution: $55^{\prime \prime}$ or $0.1 \mathrm{pc}$ ) indicates homogeneous material with a density of $3 \times 10^{3} \mathrm{~cm}^{-3}$. Clumpiness at scales around $0.01 \mathrm{pc}$ or below cannot be excluded, however.

\section{ISOCAM observations}

The ISOCAM map of the Horsehead nebula taken in the broadband filter LW2 $(5-8.5 \mu \mathrm{m})$ is presented in Fig. 2 (the map taken is the LW3 filter is comparable). The 6" pixel-field-ofview lens was used, so the Point Spread Function (PSF) is under-sampled by a factor of $\sim 2$. The observation strategy and the data processing are detailed in Abergel et al. (2002). All instrumental defects we currently understand have been corrected or flagged out. However, some remaining artefacts are visible in Fig. 2, due to memory effects still not properly corrected and which produce ghost features. For instance the bright source located at the tip of the emission produces several ghosts in the south direction at positions corresponding to the subsequent pointings of the satellite. In addition the bright filament at the edge of the Horsehead nebula produces a dark ghost at a distance of $\sim 1.5^{\prime}$ south. The photometric accuracy for the extended emission is $\sim 10 \%$, while the high frequency noise per pixel is $\sim 0.15 \mathrm{MJy} \mathrm{sr}^{-1}$. Finally, we substract the zodiacal emission estimated at the time of the observations by the model of Kelsall et al. (1998): 3.8 $\mathrm{MJy} \mathrm{sr}^{-1}$ and 25.7 $\mathrm{MJy} \mathrm{sr}^{-1}$ for the LW2 and LW3 filters, respectively.

The illuminated edge of the Horsehead nebula presents a very sharp infrared filament (Figs. 2 and 4). The spatial structure of the emission is identical for the LW2 and LW3 filters. 


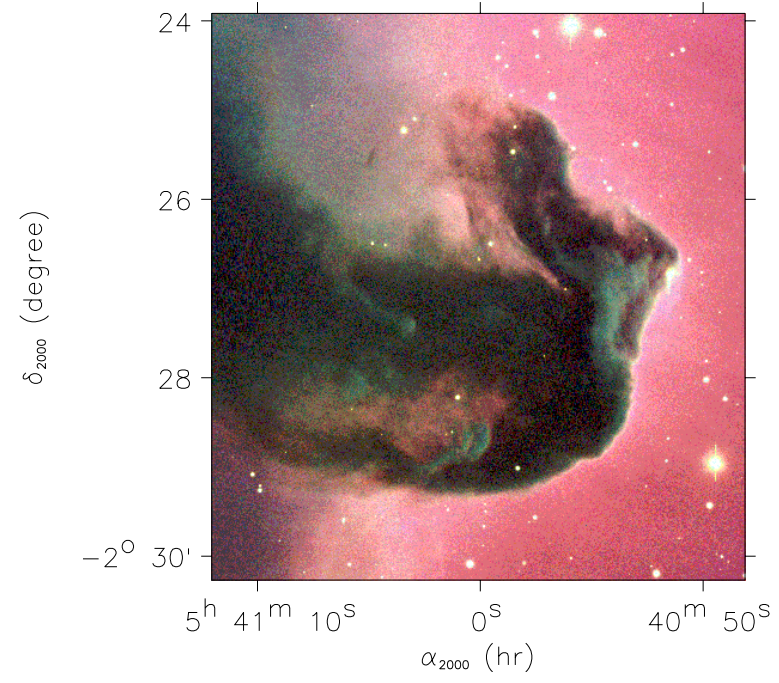

Fig. 1. Composite colour image of the Horsehead nebula with the VLT (ESO). The seeing was about $0.75^{\prime \prime}$.

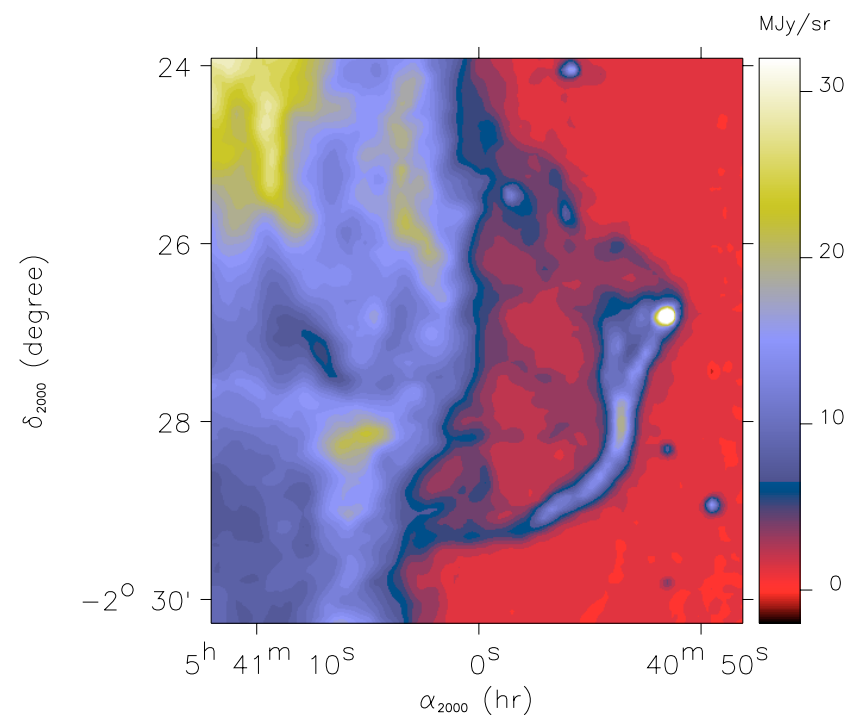

Fig. 2. ISOCAM map of the Horsehead nebula in the LW2 filter $(5-8.5 \mu \mathrm{m})$.

The filament is elongated in the north-south direction. The horizontal brightness profile along the brightest pixel of the filament is compared in Fig. 4 with the profile obtained for a point source focused at the center of a pixel and observed with the same filter and the same field of view per pixel $\left(6^{\prime \prime}\right)$. We see that the filament appears resolved, with a width around twice the pixel size $\left(\sim 10^{\prime \prime}\right.$, or $\left.\sim 0.02 \mathrm{pc}\right)$. However it is not possible to derive any precise shape because of the under-sampling of the PSF.

\section{Molecular observations}

In complement to the ISOCAM data, we conducted molecular observations at the IRAM 30-m telescope. On-the-fly maps of the $(J=1-0)$ and $(J=2-1)$ transitions of ${ }^{12} \mathrm{CO},{ }^{13} \mathrm{CO}$ and $\mathrm{C}^{18} \mathrm{O}$ were obtained in a region of $2.5^{\prime} \times 3.5^{\prime}$ for the main isotopomer, and in a more restricted band of $1.5^{\prime} \times 1^{\prime}$ for the latter ones. These maps were centred at the brightest position of

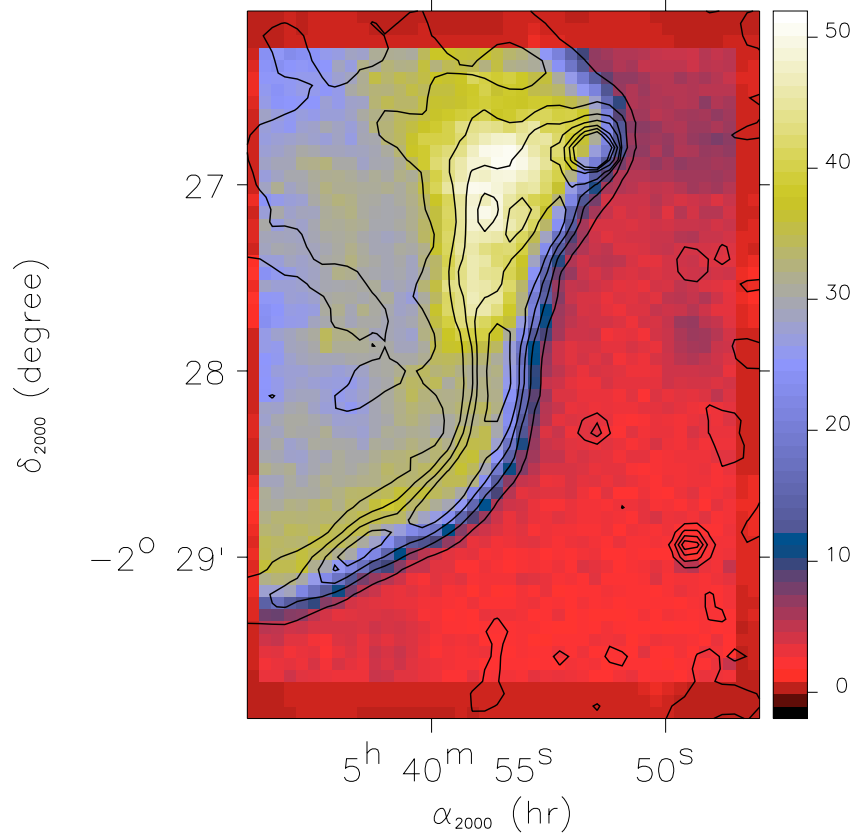

Fig. 3. ISOCAM contours of the LW2 emission (5-8.5 $\mu \mathrm{m}$, levels are 0 to 10 by 2.5 , then 10 to 25 by $5 \mathrm{MJy} / \mathrm{sr})$ plotted over the ${ }^{12} \mathrm{CO}(2-1)$ peak temperature map ( $T_{\mathrm{mb}, \mathrm{c}}$ scale, corrected for error beam pick-up).

the infrared filament. The data were acquired in January 1999. With a system temperature of order $150-250 \mathrm{~K}$ (400-500 K) and a resolution element of $80 \mathrm{kHz}$, the noise rms per regridded pixel is $0.20 \mathrm{~K}(0.35 \mathrm{~K})$ at $2.6 \mathrm{~mm}(1.3 \mathrm{~mm}$ respectively). All coverages were performed in two orthogonal directions and combined using the PLAIT algorithm developed by Emerson \& Gräve (1988), reducing significantly the spurious stripes in the maps. The calibration and conversion of the data into a corrected main beam temperature scale $\left(T_{\mathrm{mb}, \mathrm{c}}\right)$ is detailed in Appendix A.

Sample spectra obtained at the brightest position of the infrared filament are displayed in Fig. 5, while Fig. 6 presents a mosaic of spectra in the ${ }^{12} \mathrm{CO}(2-1)$ line. As mentioned by Kramer et al. (1996), the main component of the molecular emission is around $\sim 10.5 \mathrm{~km} \mathrm{~s}^{-1}$. Figure 3 shows the map of the peak temperature for the ${ }^{12} \mathrm{CO}(2-1)$ line plotted with the ISOCAM contours in the LW2 filter. Horizontal brightness profiles across the edge of the nebula are presented in Fig. 4.

\section{Structure of the edge of the Horsehead nebula}

\subsection{Interpretation of the bright infrared filament}

The bright filament detected with ISOCAM (Fig. 2) is precisely at the position of the edge of the dark nebula seen in the visible (Fig. 1) and at the edge of the molecular emission (Figs. 3, 4, 6). The border of the ${ }^{13} \mathrm{CO}$ and $\mathrm{C}^{18} \mathrm{O}$ emissions is slightly shifted to the east (Fig. 4), as expected from selective photodissociation due to self-shielding in the outermost layers of molecular structures (e.g. Fuente et al. 1993; White \& Sandell 1995). Outside the molecular cloud, the CO, ${ }^{13} \mathrm{CO}$ and $\mathrm{C}^{18} \mathrm{O}$ molecules are photodissociated, then the $\mathrm{CO}$ emission appears first, followed by the ${ }^{13} \mathrm{CO}$ emission and finally the 


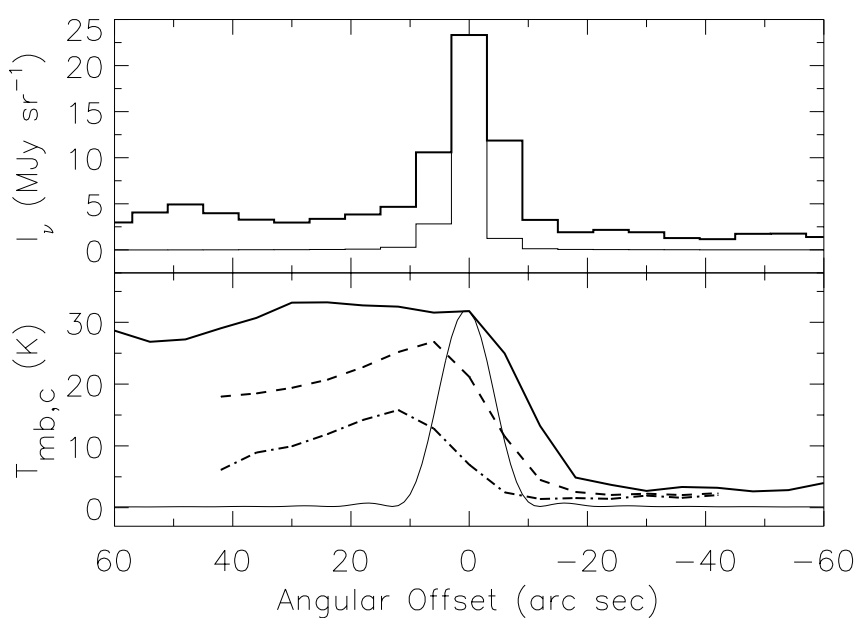

Fig. 4. Emission profiles at constant declination $\left(\delta_{2000}=-02^{\circ} 28^{\prime} 4^{\prime \prime}\right)$. The offset origin (at $\alpha_{2000}=05^{\mathrm{h}} 40^{\mathrm{m}} 53.7^{\mathrm{s}}$ ) corresponds to the ISOCAM peak on the edge of the Horsehead nebula. Upper panel: ISOCAM emission in the LW2 filter (5-8.5 $\mu \mathrm{m}$, solid thick line). The thin line shows the profile for a point source focused at the center of a pixel and observed with the same filter and the same field of view per pixel $\left(6^{\prime \prime}\right)$. Lower panel: Peak temperature for the $(J=2-1)$ transitions of ${ }^{12} \mathrm{CO}$ (solid line), ${ }^{13} \mathrm{CO}$ (dashed line) and $\mathrm{C}^{18} \mathrm{O}$ (dash-dotted line). The thin line shows the beam profile of the 30-m telescope at $1.3 \mathrm{~mm}$ (from Greve et al. 1998).

$\mathrm{C}^{18} \mathrm{O}$ emission. However the steep edge of the cloud $\left(\sim 6^{\prime \prime}\right.$ or $\sim 0.01 \mathrm{pc}$ ) is not resolved by the molecular observations (spatial resolutions are $10.5^{\prime \prime}$ and $21^{\prime \prime}$, or 0.04 and $0.02 \mathrm{pc}$, at $2.6 \mathrm{~mm}$ and $1.3 \mathrm{~mm}$ respectively).

We have seen in the introduction that, in the interstellar medium and on large angular scales, the ISOCAM emission in the LW2 filter scales, to a first order, with the incident radiation field. Unfortunately, we have no observation of an independant tracer of the column density taken at a sufficient angular resolution to relate the spatial variations of the infrared emission observed at small angular scale to variations of the abundance or the optical properties of the aromatic emitters. Therefore, in this paper we work in the hypothesis that the LW2 emission is proportional to the column density and the incident radiation field. The infrared extinction is only significant for very large column densities (typically $A_{V}=50-100$ ). Using the emissivity of the aromatic particles in the LW2 filter found by Habart et al. (2003) in the $\rho$ Ophiuchi PDR, we can estimate the column density of the infrared emitting region at the edge of the nebula from the peak emission of the infrared profile ( $20 \mathrm{MJy} \mathrm{sr}^{-1}$, see Fig. 4). We obtain $A_{V} \sim 2$, so we can neglect the effects of the infrared extinction. The steep increase of the infrared emission on the illuminated side (negative offsets in Fig. 4) is due to a steep increase of the column density (size less than $10^{\prime \prime}$ or $0.02 \mathrm{pc}$ ), while on the other side (positive offsets in Fig. 4) the decrease of the emission is due to the extinction of the incident radiation by dense material. The infrared emission emerging from the inner parts of the dark nebula (positive offsets) is $\sim 10$ times lower than the emission of the bright filament. This fact suggests that the dense material forming the edge of the Horsehead nebula is illuminated edge-on by $\sigma$ Ori, so that the particles behind the border are

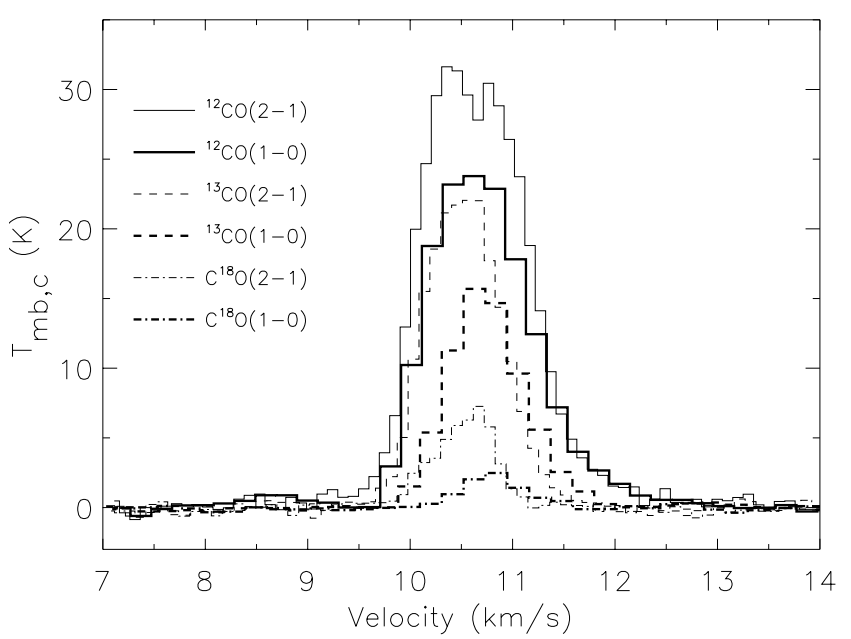

Fig. 5. Spectra of the three isotopemers in the two observed positions ( $J=1-0$ thick, $J=2-1$ thin) at the position corresponding to the offset origin of Fig. 4.

not heated by the incident radiation field. The dense material forming the edge of the Horsehead nebula and $\sigma$ Ori are in a common plane likely near the plane of the sky, since the density gradient, as reflected by the brightness gradient at the illuminated edge (negative offsets in Fig. 4), is extremely steep (below $\left.10^{\prime \prime}\right)$. A large angle $\left(\sim 30^{\circ}\right.$ or more) between this common plane and the plane of the sky would produce smoother brightness gradients on both sides of the peak.

To the west of the Horsehead nebula the LW2 emission is fairly uniform $\left(1.7 \pm 0.5 \mathrm{MJy} \mathrm{sr}^{-1}\right)$ and corresponds to the minimal emission measured by ISOCAM within the field taken in the southern part of Orion B. This emission could be due to particles in the low density HII region facing the illuminated edge of the nebula (Sect. 5.4). In the LW3 filter, the detection of faint uniform emission is not possible because of the amplitude of the zodiacal emission.

\subsection{Estimate of the density from the molecular data}

In the optically thick assumption, it is well known (e.g. Bergin et al. 1994) that the observed excitation temperatures provide a very good estimate of the kinetic temperatures. For an observed ${ }^{12} \mathrm{CO}(1-0)$ radiation temperature $T_{\mathrm{R}}$, this simply writes:

$$
T_{\mathrm{k}} \simeq \frac{5.54 K}{\ln \left[5.54 /\left(T_{\mathrm{R}}+0.87\right)+1\right]}
$$

In the Horsehead nebula, the inferred kinetic temperatures fall in the range $30-40 \mathrm{~K}$. Assuming that all three isotopomers have the same kinetic temperature, we have used these temperatures to study the $(J=1-0)$ and $(J=2-1) \mathrm{C}^{18} \mathrm{O}$ emissions, performing Large Velocity Gradient (LVG) calculations along directions of interest. These emissions are optically thin, and are consistent with densities in the range $1-4 \times 10^{4} \mathrm{~cm}^{-3}$, with no significant trend in the probed area. This is comparable to the local densities reported in previous papers (Zhou et al. 1993; Kramer et al. 1996). We note however that the densities found here should be seen as lower limits since the kinetic temperature in the shielded $\mathrm{C}^{18} \mathrm{O}$ layers could be lower than in the outer 


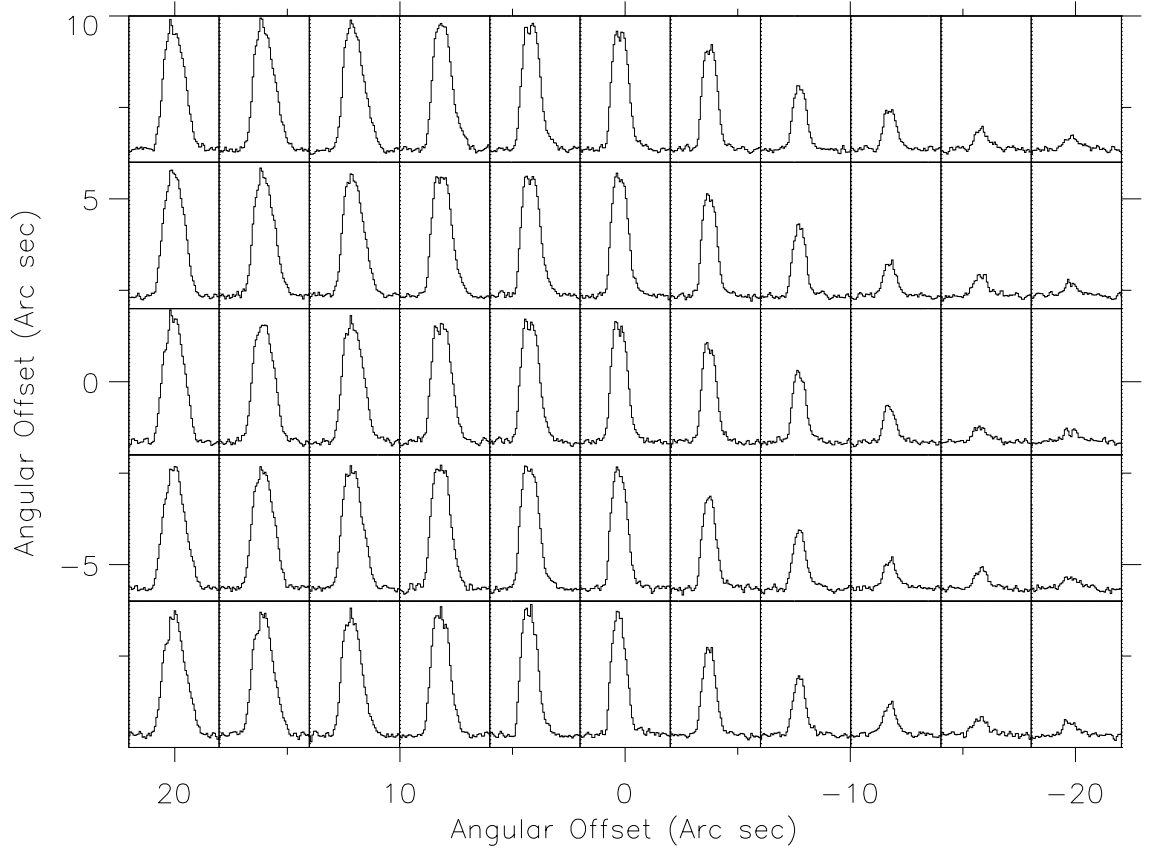

Fig. 6. Mosaic of spectra in the ${ }^{12} \mathrm{CO}(2-1)$ line. The offset origin is the same as Fig. 4. Each spectrum is shown on a $4^{\prime \prime}$ grid with $8.5 \mathrm{~km} \mathrm{~s}{ }^{-1}<$ $V_{\mathrm{LSR}}<13.5 \mathrm{~km} \mathrm{~s}^{-1}$ and $-3 \mathrm{~K}<T_{\mathrm{mb}, \mathrm{c}}<35 \mathrm{~K}$.

layers traced by $\mathrm{CO}$. The corresponding molecular column densities are of order $3-6 \times 10^{15} \mathrm{~cm}^{-3}$, which is comparable to column densities reported in the Orion bar (White \& Sandell 1995) and in NGC 7023 (Fuente et al. 1993). However, accurate comparisons with fractional abundances found in other PDRs still await independent measurements of the total $\mathrm{H}_{2}$ column density. Moreover, we have seen that the edge of the molecular emission is not resolved by the 30-m data. Therefore unavoidable smearing effects preclude any estimate of the density at the edge of the nebula. The value of $1-4 \times 10^{4} \mathrm{~cm}^{-3}$ we have derived from the data corresponds to material beyond the edge.

\subsection{Local density behind the infrared filament}

The density behind the illuminated edge can be estimated from the penetration depth of the incident radiation deduced from the infrared brightness profile (Fig. 4). We consider a very simple model, where the local density and the column density are constant inside the dark cloud, and negligible outside. Let $I_{0, \mathrm{UV}}$ be the incident radiation field. At a distance $z$ from the edge, this radiation writes: $I(z)_{\mathrm{UV}}=I_{0, \mathrm{UV}} \mathrm{e}^{-K z}, K$ being the UV extinction coefficient of the dust. The ISOCAM emission in the LW2 filter scales, to a first order, with the incident radiation field, so it can be written: $I(z)=I_{0} \mathrm{e}^{-K z}$, where $I_{0}$ is the peak emission of the infrared filament. The penetration depth is $\sim 10^{-2} \mathrm{pc}\left(\sim 6^{\prime \prime}\right)$, which corresponds to $K \sim 100 \mathrm{pc}^{-1}$. Using standard conversion numbers $\left(\tau_{\mathrm{UV}} / \tau_{V}=2.8\right.$ and $A_{V} / E_{B-V}=$ 3.1 from Savage \& Mathis $1979, \tau_{V} / A_{V}=1 / 1.086, N_{\mathrm{H}} / E_{B-V}=$ $5.9 \times 10^{21} \mathrm{~cm}^{-2}$ from Bohlin et al. 1978), we obtain:

$K=n \times \frac{\tau_{\mathrm{UV}}}{\tau_{V}} \frac{\tau_{V}}{A_{V}} \frac{A_{V}}{E_{B-V}} \frac{E_{B-V}}{N_{\mathrm{H}}}=n \times 410^{-3} \mathrm{pc}^{-1}$

where the local density $n$ is in units of $\mathrm{cm}^{-3}$.
From $K \sim 100 \mathrm{pc}^{-1}$, we finally infer $\mathrm{n} \sim 2 \times 10^{4} \mathrm{~cm}^{-3}$, which is comparable to the value estimated from our molecular observations beyond the illuminated edge (for distances of $10-30^{\prime \prime}$ from the peak position of the infrared filament). In our simple model, we have assumed an infinite gradient of column density at the illuminated edge, which is obviously a simplifying assumption. The infrared profile (Fig. 4) is compatible with a steep edge $\left(\sim 6^{\prime \prime}\right.$ or $\left.\sim 0.01 \mathrm{pc}\right)$, but geometrical effects can obviously smooth the emerging profile. In any case, the value of $\sim 2 \times 10^{4} \mathrm{~cm}^{-3}$ determined from the extinction depth of UV radiations can only be considered as a lower limit for homogeneous material.

The density of the neutral region exposed to the ionizing radiations, just behind the ionization front, can be estimated from the flux of ionizing photons. Details are given in Appendix B. We find a value $\left(\sim 0.8 \times 10^{4} \mathrm{~cm}^{-3}\right)$ comparable to the density measured behind the infrared filament from the penetration depth of UV radiation $\left(\sim 2 \times 10^{4} \mathrm{~cm}^{-3}\right)$. However, these two densities have not to be equal, since the ionization front corresponds to the most external regions of the cloud, while UV radiations heating the aromatic particles are absorbed deeper inside the cloud.

\subsection{Infrared emission for the HII region}

Different authors (e.g. Crété et al. 1999) have suggested that the relative abundance of aromatic particles strongly decreases in HII regions with high incident radiation fields $\left(\chi \sim 10^{4}-10^{5}\right)$. Can we find a comparable effect in the HII region facing the Horsehead nebula?

Let $n_{1}$ and $n_{2}$ (and $T_{1}$ and $T_{2}$ ) be the densities (and temperatures) in the neutral and HII regions behind and ahead the ionization front, respectively. From Eqs. (B.2) and (B.4), and 
using $T_{1} \sim 300 \mathrm{~K}$ and $T_{2} \sim 10^{4} \mathrm{~K}$ (Appendix B), we have: $n_{1} / n_{2}=(\gamma+1) T_{2} / T_{1} \sim 50$. From this ratio and the value of the peak emission of the LW2 profile $\left(\sim 20 \mathrm{MJy} \mathrm{sr}^{-1}\right)$, we can estimate the LW2 emission from the HII region ahead the ionization front assuming constant abundance and emissivity of the emitting particles across the interface. We obtain a value of $\sim 20 / 50=0.4 \mathrm{MJy} \mathrm{sr}^{-1}$, which is comparable to the emission detected in front of the edge $\left(1.7 \pm 0.5 \mathrm{MJy} \mathrm{sr}^{-1}\right)$. We see that we cannot evidence any decrease of the abundance or the emissivity of the aromatic particles from the neutral to the HII regions. The same conclusion was obtained by Habart et al. (2003) for the interface of the $\rho$ Ophiuchi main cloud illuminated, as the Horsehead nebula, by a moderate radiation field $(\chi \sim 400)$. We have also shown in Abergel et al. (2002) that the contribution of the aromatic bands relative to intensity of the underlying continuum emission is systematically higher in low density HII regions than at the illuminated edges of dense structures (for $\chi \sim 100-1000$ ). These results indicate that the aromatic particles are not systematically destroyed in HII regions when the radiation field is limited $(\chi<1000)$.

\subsection{Clumpiness}

The material at the illuminated edge could also be nonhomogeneous on scales smaller than the upper limit of the penetration depth $\left(10^{-2} \mathrm{pc}\right)$. In order to constrain this small scale structure, we have estimated the effective extinction of non-homogeneous material using the approach of Meixner \& Tielens (1993) based on the formalism of Boissé (1990) for two-phase clumpy regions (see Appendix $\mathrm{C}$ for details). In this approach, the clumping is described by three parameters: the average density, the density contrast between clumps and interclump material and the filling factor. The clump size is much smaller than the penetration depth of $10^{-2} \mathrm{pc}$.

Clumping reduces the effective extinction and thus increases the observed penetration depth. This is quantitatively illustrated in Fig. C.3. For example, for an average density of $2 \times 10^{6} \mathrm{~cm}^{-3}$, the presence of clumps with typical sizes of $10^{-4}-10^{-3} \mathrm{pc}$ with a volume filling factor of about 0.1 , clumping reduces the extinction by a factor about 100 (Fig. C.3) and the penetration depth is the same that for homogeneous medium with a density of $2 \times 10^{4} \mathrm{~cm}^{-3}$. This is due to the decreasing contribution of the inter-clump medium to the extinction. Thus, the presence of clumps with sizes smaller than $\sim 10^{-3}$ pc cannot be excluded. In that case the density derived from our $\mathrm{C}^{18} \mathrm{O}$ observations (a few $10^{4} \mathrm{~cm}^{-3}$, see Sect. 5.2) could correspond to the inter-clump material. A comparable "interclump" density is also observed in the PDR of the Orion bar together with dense clumps with size in the range 0.02 to $0.1 \mathrm{pc}$ (van der Werf et al. 1996).

\section{Conclusions}

The illuminated edge of the Horsehead nebula presents a very sharp infrared filament (width $\sim 10^{\prime \prime}$, or $\sim 0.02 \mathrm{pc}$ ), likely the sharpest infrared filament detected in our Galaxy by ISOCAM. The increase of emission on the illuminated side is due to a steep increase of column density, while on the other side the decrease of the emission is due to the extinction of the incident radiation by dense material. The most straightforward explanation is that most of the dense matter is within a flat structure illuminated edge-on by $\sigma$ Ori. Moreover, this structure is very likely located within a plane close to the plane of the sky. This very specific three-dimensional shape could result from the recent history of the nebula. The edge-on geometry may be due to the presence of dense filaments inside the parental cloud which have shielded the material located in its shadow from the photoevaporating radiations emitted by $\sigma$ Ori. A comparable scenario to explain the formation of columns of gas that have not evaporated due to shadowing behind dense clumps at their head has also been suggested by Hester et al. (1996) from HST observations of M 16. As suggested recently by Pound et al. (2003), the dense filaments could be pre-existing structures in the parental cloud, or results from instabilities at the ionization front.

At the illuminated edge of the Horsehead nebula the incident radiation appears absorbed within a thin layer (thickness below $\sim 6^{\prime \prime}$ or $\sim 10^{-2} \mathrm{pc}$ ). Within this layer located just behind the infrared filament, and assuming homogeneous material, the density must be at least of $\sim 2 \times 10^{4} \mathrm{~cm}^{-3}$, which corresponds to the density deduced beyond the edge from our $\mathrm{C}^{18} \mathrm{O}$ observations. However the absorbing material may also be non homogeneous, with clump sizes significantly smaller than $10^{-2} \mathrm{pc}$. In that case, the density derived from the $\mathrm{C}^{18} \mathrm{O}$ observations could correspond to the "interclump" material. The average density behind the infrared filament must in any case be higher than $\sim 2 \times 10^{4} \mathrm{~cm}^{-3}$. It is interesting to note that this lower limit is also comparable to the density of the neutral material just behind the ionization front, estimated to $0.8 \times 10^{4} \mathrm{~cm}^{-3}$ in the stationary case. Finally we cannot evidence any drecrease of the abundance or the emissivity of the aromatic particles from the neutral to the HII regions of the Horsehead nebula, suggesting that the aromatic particles are not systematically destroyed in HII regions when the radiation field is limited $(\chi<1000)$.

ISOCAM observations bring new constraints on the shape of the Horsehead nebula, but the angular resolution of 6" is still unsufficient to resolve properly the density structure. We will present in forthcoming papers a combination of interferometric observations taken at the Plateau de Bures with the 30-m data, and high resolution mapping of the 1-0 S(1) fluorescent line of $\mathrm{H}_{2}$.

Acknowledgements. ISO is an ESA project with instruments funded by ESA Member States and with the participation of ISAS and NASA. The authors would like to thank F. Bensch and J.-F. Panis for constructive discussions on the error beam removal technique.

\section{Appendix A: Calibration and beam correction of the molecular observations}

The data were first calibrated to the antenna temperature $\left(T_{\mathrm{A}}^{*}\right)$ scale using the chopper wheel method (Penzias \& Burrus 1973). In order to take into account the extended emission picked up by the error beam of the 30-m telescope (Greve et al. 1998), we used the KOSMA data published by Kramer et al. (1996) for the ${ }^{12} \mathrm{CO}$ and ${ }^{13} \mathrm{CO} J=2-1$ lines, and applied 
the error beam removal technique presented by Falgarone et al. (1998) and Bensch et al. (2001). This method allows for each spatial pixel and each velocity channel an independent correction of the main beam temperature $\left(T_{\mathrm{mb}}\right)$. Due most probably to the under-sampling of the KOSMA data, the technique however proved inefficient in the line wings and we preferred not to apply the correction on a channel-to-channel basis. Instead, we used the approximation introduced by Teyssier et al. (2002), found to be consistent with the median correction factor deduced from the error beam removal technique. This correction applies equally to all pixels and velocity channels of the considered field. For $\mathrm{C}^{18} \mathrm{O}(2-1)$ where no KOSMA data are available, we assumed a similar spatial distribution as ${ }^{13} \mathrm{CO}(2-1)$ and thus similar conversion factors. No equivalent data could be found at $2.6 \mathrm{~mm}$. The error beam contribution is however less significant at this wavelength (Greve et al. 1998) and we applied a scaling factor based on the approximation introduced above.

In comparison to what would be obtained in a $T_{\mathrm{mb}}$ scale, the final temperatures are reduced by $20 \%$ for ${ }^{12} \mathrm{CO}(2-1), 12 \%$ for ${ }^{13} \mathrm{CO}(2-1)$ and $\mathrm{C}^{18} \mathrm{O}(2-1)$, and $5 \%$ for the $2.6 \mathrm{~mm}$ transitions. This also shows that our assumption of a comparable ${ }^{13} \mathrm{CO}$ and $\mathrm{C}^{18} \mathrm{O}$ spatial distribution could lead to underestimate the $\mathrm{C}^{18} \mathrm{O}(2-1)$ line intensities by at most $\sim 10 \%$, which has no significant consequences on the results presented thereafter. Following Bensch et al. (2001), we call the corrected main beam temperature scale $T_{\mathrm{mb}, \mathrm{c}}$.

\section{Appendix B: Local density behind the ionization front}

In this Appendix, the subscripts " 1 " and " 2 " correspond to the neutral and HII regions behind and ahead the ionization front, respectively. $T_{1}$ and $T_{2}$ are the temperatures, $n_{1}$ and $n_{2}$ the gas densities, $V_{1}$ and $V_{2}$ the velocities relative to the front and in a direction perpendicular to the front. In the hypothesis that the gas in the HII region ahead the ionization front expands freely, $V_{2}$ is equal to the sound speed $c_{2}=\sqrt{\frac{\gamma k T_{2}}{\mu_{2} m_{\mathrm{H}}}} \sim 10 \mathrm{~km} \mathrm{~s}^{-1}$ (see below). The average mass per atom in the neutral and HII regions is $\mu_{1} m_{\mathrm{H}}$ and $\mu_{2} m_{\mathrm{H}}$, respectively, with $\mu_{1}=1.4$ and $\mu_{2}=0.7$.

We assume that $\sigma$ Ori is at a distance $r_{\mathrm{S}}=3.5 \mathrm{pc}$ from the edge of the Horsehead nebula, corresponding to the angular distance of $0.5^{\circ}$. As explained by Spitzer (1978), the protons produced at the ionization front are at a higher pressure than the gas outside the cloud. Thus the ionized gas expands toward the star, forming a thin layer of dense ionized gas wrapping the neutral cloud. The flux of ionizing photons reaching this layer is: $J_{0}=S_{0} \mathrm{e}^{-\tau} / 4 \pi r_{\mathrm{S}}^{2} \sim 6.4 \times 10^{8} \mathrm{~cm}^{-2} \mathrm{~s}^{-1}$, where $S_{0}$ is the number of ionizing photons emitted by the star $\left(10^{48.25}\right.$ for a $\mathrm{O} 9.5 \mathrm{~V}$ star, from Schaerer \& de Koter 1997), and $\mathrm{e}^{-\tau}$ the extinction of the Lyman photons by dust in the HII region, taken equal to 0.5 . Electrons will recombine with protons in the layer of dense ionized gas, and the resultant $\mathrm{H}$ atoms reionized, which reduce the flux of photons reaching the ionization front, $J$. In a spherical geometry, Spitzer (1978) has shown that the ratio $J / J_{0}$ can be estimated with:

$$
\frac{2 J_{0}}{J}=1+\left(1+\frac{4 \alpha J_{0} R_{i}}{3 c_{2}^{2}}\right)^{1 / 2} \text {. }
$$

Here $\alpha$ is the recombinaison coefficient and $R_{i}$ the radius of curvature of the ionization front. With $\alpha=4 \times 10^{-13} \mathrm{~cm}^{-3} \mathrm{~s}^{-1}$, $R_{i}=0.2 \mathrm{pc}, c_{2}=10 \mathrm{~km} \mathrm{~s}^{-1}$ and the value of $J_{0}$ given above, we obtain $J=J_{0} / 8=0.8 \times 10^{8} \mathrm{~cm}^{-2}$.

Following Kaplan (1966), the velocity and the density in the neutral region $\left(V_{1}\right.$ and $\left.n_{1}\right)$ can be estimated in the stationary case using the mass and momentum conservation equations across the ionization front which write:

$\mu_{1} J=\mu_{1} n_{1} \times V_{1}=\mu_{2} n_{2} \times V_{2}$

$\mu_{1} m_{\mathrm{H}} n_{1}\left[\frac{k T_{1}}{\mu_{1} m_{\mathrm{H}}}+V_{1}^{2}\right]=\mu_{2} m_{\mathrm{H}} n_{2}\left[\frac{k T_{2}}{\mu_{2} m_{\mathrm{H}}}+V_{2}^{2}\right]$.

Equation (B.3) also contains a radiation pressure term, but it is negligible. Finally, in the case of a rarefaction ionization front $\left(n_{2}<n_{1}\right)$, we obtain from these two equations:

$\frac{V_{1}}{V_{2}}=\frac{1}{\gamma+1} \frac{\mu_{2}}{\mu_{1}} \frac{T_{1}}{T_{2}}$.

From Eqs. (B.2) and (B.4), we obtain finally:

$n_{1}=J(\gamma+1) \frac{1}{\mu_{2}} \frac{T_{2}}{T_{1}} \sqrt{\frac{\mu_{2} m_{\mathrm{H}}}{\gamma k T_{2}}}$

The temperature in the HII region, $T_{2}$, can be estimated from the equation for conservation of energy (Kaplan 1966). Values close to $10^{4} \mathrm{~K}$ are generally obtained, so we take $T_{2}=10^{4} \mathrm{~K}$ (which gives $c_{2} \sim 10 \mathrm{~km} \mathrm{~s}^{-1}$ ). For the neutral region, we take $T_{1}=300 \mathrm{~K}$ (value inferred from $\mathrm{H}_{2}$ excitation in PDRs, e.g. Habart et al. 2003). Finally, we obtain, in the neutral region just behind the ionization front: $n_{1} \sim 0.8 \times 10^{4} \mathrm{~cm}^{-3}$.

\section{Appendix C: Effects of the clumpiness}

We follow the approach of Meixner \& Tielens (1993) based on the radiative transfer formalism developped by Boissé (1990) for two-phase clumpy regions. The physical processes considered here are the absorption and isotropic scattering by dust grains. The radiative transfer in a clumpy region is reduced to that of a homogeneous region characterized by an effective extinction coefficient $K_{\mathrm{e}}$ and an albedo $\omega_{\mathrm{e}}$ (Eqs. (26) and (27) of Boissé 1990), both of them decreasing with the clumpiness. Moreover, the spatial variations of the incident intensity $\phi^{+}(z)$ with the depth $z$ are nearly exponential:

$\phi^{+}(z) \simeq \phi^{+}(0) \exp \left(-K_{\mathrm{r}} z\right)$

with:

$K_{\mathrm{r}}=\sqrt{\frac{\left(1-\omega_{\mathrm{e}}\right)}{\left(1-\frac{2 \omega_{\mathrm{e}}}{3}\right)}} K_{\mathrm{e}}$

The average density writes: $n_{\text {avg }}=p_{\mathrm{c}} n_{\mathrm{c}}+p_{\text {ic }} n_{\mathrm{ic}}$, where $p_{\mathrm{c}}$, $p_{\text {ic }}, n_{\mathrm{c}}, n_{\mathrm{ic}}$ are the volume filling factors and densities of the 


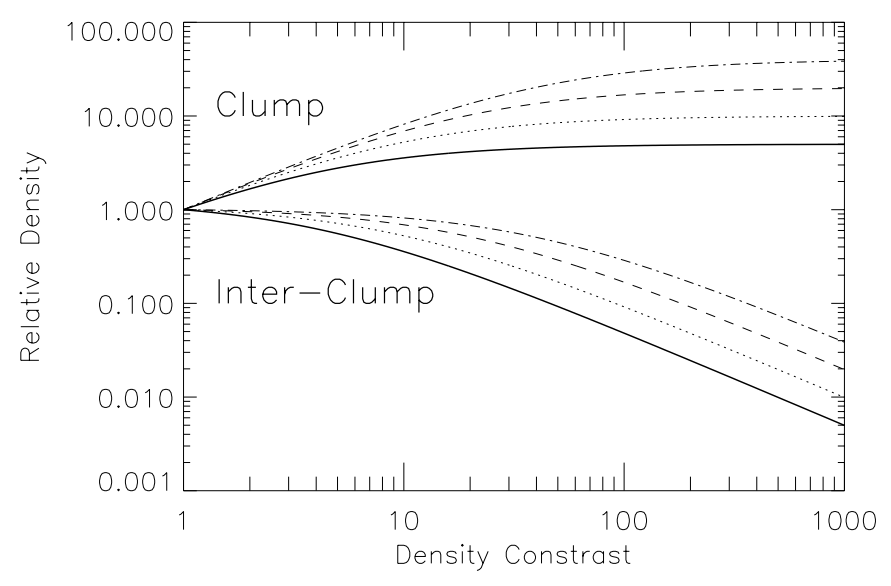

Fig. C.1. Clump and inter-clump relative densities $\left(n_{\mathrm{c}} / n_{\mathrm{avg}}\right.$ and $n_{\text {ic }} / n_{\text {avg }}$, respectively) vs. the density contrast $d_{\mathrm{c} / \text { ic }}$ for different filling factors: $p_{\mathrm{c}}=0.2$ (solid line), 0.1 (dotted line), 0.05 (dashed line), 0.025 (dashed-dotted line).

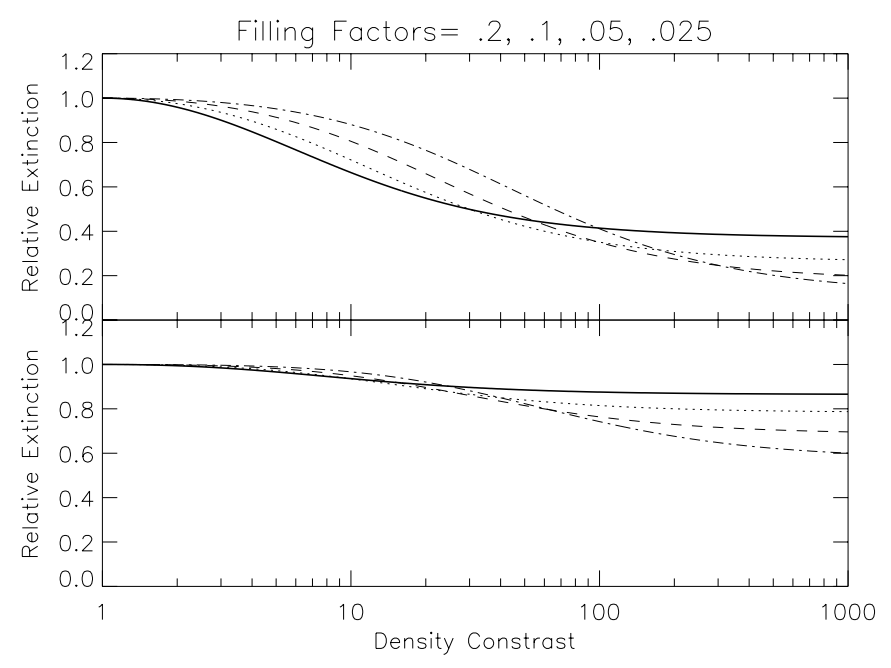

Fig. C.2. Relative value of the effective extinction coefficient $K_{\mathrm{r}}$ vs. the density contrast $d_{\mathrm{c} / \mathrm{ic}}$ for different filling factors: $p_{\mathrm{c}}=0.2$ (solid line), 0.1 (dotted line), 0.05 (dashed line), 0.025 (dashed-dotted line). The point at $(1,1)$ corresponds to the homogeneous case. The average density is $n_{\text {avg }}=2 \times 10^{4} \mathrm{~cm}^{-3}$. Top: For $M_{\mathrm{c}, \text { avg }}=10^{-3} M_{\odot}$, Clump diameters: $4.5 \times 10^{-3}-1.5 \times 10^{-2} \mathrm{pc}$. Bottom: For $M_{\mathrm{c}, \mathrm{avg}}=10^{-6} M_{\odot}$, Clump diameters: $4.5 \times 10^{-4}-1.5 \times 10^{-3} \mathrm{pc}$. The presence of very small clumps does not significantly affect the effective extinction coefficient.

clumps and inter-clump material, respectively $\left(p_{\mathrm{c}}+p_{\text {ic }}=1\right)$. The fraction of the mass within the clumps is: $f_{\mathrm{c}}=p_{\mathrm{c}} \times n_{\mathrm{c}} / n_{\mathrm{avg}}$. The clumpiness is measured using the density contrast between clumps and inter-clump material: $d_{\mathrm{c} / \text { ic }}=n_{\mathrm{c}} / n_{\mathrm{ic}}$. In Fig. C. 1 is presented the variations of clump and inter-clump densities, for different values of the filling factor.

In practice, we first assign the average density $n_{\text {avg }}(2 \times$ $10^{4} \mathrm{~cm}^{-3}$ or $\left.2 \times 10^{6} \mathrm{~cm}^{-3}\right)$, the average clump mass $M_{\mathrm{c}, \text { avg }}\left(10^{-3}\right.$ or $10^{-6} M_{\odot}$ ) and the filling factor $p_{\mathrm{c}}$ (from 0.2 to 0.025 ). For an average density $n_{\text {avg }}=2 \times 10^{4} \mathrm{~cm}^{-3}$, an average clump mass $M_{\mathrm{c}, \text { avg }}$ of $10^{-3} M_{\odot}$ (resp. $10^{-6} M_{\odot}$ ) implies clump diameters in the range $4.5 \times 10^{-3}-1.5 \times 10^{-2} \mathrm{pc}$ (resp. $4.5 \times 10^{-4}-1.5 \times$ $10^{-3} \mathrm{pc}$ ) depending on the density contrast. We then compute the effective extinction coefficient $K_{\mathrm{r}}$ for different values of the

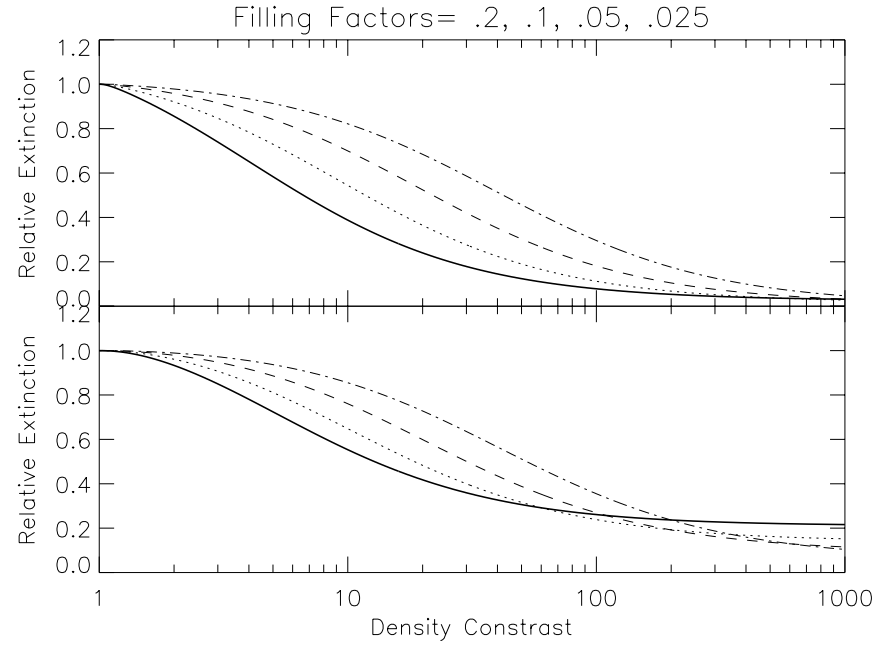

Fig. C.3. Same as Fig. C. 2 for an average density $n_{\text {avg }}=2 \times 10^{6} \mathrm{~cm}^{-3}$. Top: For $M_{\mathrm{c}, \text { avg }}=10^{-3} M_{\odot}$, Clump diameters: $1 \times 10^{-3}-3.5 \times 10^{-3} \mathrm{pc}$. Bottom: For $M_{\mathrm{c}, \text { avg }}=10^{-6} M_{\odot}$, Clump diameters: $1 \times 10^{-4}-3.5 \times$ $10^{-4} \mathrm{pc}$.

density contrast. In Boissé (1990), the clumps have an exponential size distribution, $\exp \left(-l / l_{0}\right)$, where $l_{0}$ is the average length through a clump (for spherical clumps, $l_{0}=4 r_{\mathrm{c}} / 3, r_{\mathrm{c}}$ being the average clump radius).

Figure C.2 (resp. Fig. C.3) presents a set of results for an average density of $2 \times 10^{4} \mathrm{~cm}^{-3}$ (resp. $2 \times 10^{6} \mathrm{~cm}^{-3}$ ) and average clump masses $M_{\mathrm{c} \text {,avg }}$ of $10^{-3} M_{\odot}$ and $10^{-6} M_{\odot}$. We see that, for density constrats below 100 , the extinction coefficient $\left(K_{\mathrm{r}}\right)$ significantly decreases for increasing density contrast and filling factor, as expected. For very large density contrasts $\left(d_{\mathrm{c} / \mathrm{ic}}>100\right)$, the extinction is purely geometrical and essentially due to optically thick clumps. Therefore the computed values of $K_{\mathrm{r}}$ converge towards an asymptotic value $K_{\mathrm{r},+\infty}$ which decreases with decreasing filling factors $p_{\mathrm{c}}$.

\section{References}

Abergel, A., André, P., Bacmann, A., et al. 1999, Spatial distribution of dust from cirrus to dense clouds, in The Universe as seen by ISO, ed. P. Cox, \& M. F. Kessler (Noordwijk: ESA Pub.), SP 427, 2,615

Abergel, A., Bernard, J.-P., Boulanger, F., et al. 2002, A\&A, 389, 239 Anthony-Twarog, B. J. 1982, AJ, 87, 1213

Barnard, E. E. 1913, ApJ, 38, 496

Bensch, F., Panis, J.-F., Stutzki, J., et al. 2001, A\&A, 365, 275

Bergin, E. A., Goldsmith, P. F., Snell, R. L., et al. 1994, ApJ, 431, 674

Boissé, P. 1990, A\&A, 228, 483

Bohlin, R. C., Savage, B. D., \& Drake, J. F. 1978, ApJ, 224, 132

Boulanger, F., Abergel, A., Bernard, J.-P., et al. 1998, The Nature of Small Interstellar Dust Particles, in Star Formation with ISO, ed. J. L. Yun, \& R. Liseau (San Francisco: Astronomical Society of the Pacific), 15

Cesarsky, C. J., Abergel, A., Agnèse, P., et al. 1996, A\&A, 315, L32

Crété, E., Giard, M., Joblin, C., et al. 1999, A\&A, 352, 277

Emerson, D. T., \& Gräve, R. 1988, A\&A, 190, 353

Falgarone, E., Panis, J.-F., Heithausen, A., et al. 1998, A\&A, 331, 669

Field, D., Lemaire, J. L., Pineau des Forêts, G., et al. 1998, A\&A, 333, 280 
Fuente, A., Martin-Pintado, J., Cernicharo, J., \& Bachiller, R. 1993, A\&A, 276, 473

Greve, A., Kramer, C., \& Wild, W. 1998, A\&AS, 133, 271

Habart, E., Boulanger, F., Verstraete, L., et al. 2003, A\&A, 397, 623

Habing, H. J. 1968, Bull. Astr. Netherlands, 19, 421

Hester, J. J., Scowen, P. A., Sankrit, R., et al. 1996, AJ, 111, 2349

Hollenbach, D. J., \& Tielens, A. G. G. M. 1997, ARA\&A, 35, 179

Kaplan, S. A. 1966, Interstellar Gas Dynamics, ed. F. D. Kahn (Pergamon Press Oxford)

Kelsall, T., Weiland, J. L., Franz, B. A., et al. 1998, ApJ, 508, 25

Kramer, C., Stutzki, J., \& Winnewisser, G. 1996, A\&A, 307, 915

Lada, E. A., Evans, N. J., Depoy, D. L., et al. 1991, ApJ, 371, 171

Léger, A., \& Puget, J. L. 1984, A\&A, 137, L5

McCartney, M. S. K., Brand, P. W. J. L., Burton, M. G., et al. 1999, MNRAS, 307, 315

Malin, D. 1987, Sky \& Telescope, 74, 253

Meixner, M., \& Tielens, A. G. G. M. 1993, ApJ, 405, 216

Moore, E. M., \& Marscher, A. P. 1995, ApJ, 452, 671

Panagia, N. 1973, AJ, 78, 929

Penzias, A. A., \& Burrus, C. A. 1973, ARA\&A, 11, 51
Perryman, M. A. C., Lindegren, L., Kovalevsky, J., et al. 1997, A\&A, 323,49

Pound, M. W., Reipurth, B., \& Bally, J. 2003, AJ, 125, 2108

Reipurth, B., \& Bouchet, P. 1984, A\&A, 137, L1

Savage, B. D., \& Mathis, J. S. 1979, ARA\&A, 17, 73

Sellgren, K., Allamandola, L. J., Bregman, J. D., et al. 1985, ApJ, 299, 416

Schaerer, D., \& de Koter, A. 1997, A\&A, 322, 598

Spitzer, L. Jr. 1978, Physical processes in the interstellar medium (New York: Wiley-Interscience)

Teyssier, D., Hennebelle, P., \& Pérault, M. 2002, A\&A, 382, 624

Tielens, A. G. G. M., Meixner, M. M., van der Werf, P. P., et al. 1993, Nature, 262, 86

Uchida, K. I., Sellgren, K., Werner, M. W., et al. 2000, ApJ, 530, 817 Van der Werf, P. P., Stutzki, J., Sternberg, A., \& Krabbe, A. 1996, A\&A, 313, 633

Warren, W. H. Jr., \& Hesser, J. E. 1977, ApJS, 34, 115

White, G. J., \& Sandell, G. 1995, A\&A, 299, 179

Zhou, S., Jaffe, D. T., Howe, J. E., et al. 1993, ApJ, 419, 190 